\title{
Influence of Flexible Substrate Materials on the Performance of Polymer Composite Gas Sensors
}

\author{
Thomas Kinkeldei, Christoph Zysset, Niko Münzenriederand Gerhard Tröster \\ Wearable Computing Lab, Swiss Federal Institute of Technology (ETH), Zurich, Switzerland, \\ Kinkeldei@ife.ee.ethz.ch
}

\begin{abstract}
:
We report on the fabrication of gas sensors on different polymer substrates to evaluate the substrate material influence on the gas sensor performance. For this purpose, we developed a resistive type gas sensor out of a conductive polymer composite material on flexible polymer substrates (PI, PEN, PPS and $\mathrm{PEI}$ ). We compared the sensors on polymer substrates to a similar one on a glass substrate as ground truth. The exposure to increasing levels of humidity showed an effect on the gas sensing behavior on plastic substrates. At a jump from $0 \% \mathrm{RH}$ to $60 \% \mathrm{RH}$, PEI substrates showed the lowest substrate influence with resistance changes of about $0.2 \%$. At the same conditions PEN and PPS substrates showed substrate influences with resistance changes of about $1 \%$. An exposure to 1000 ppm acetone in dry air showed a similar performance among all substrates.
\end{abstract}

Key words: flexible electronics, gas sensor, carbon black, conductive polymer, substrate influence

\section{Introduction}

A recent development in electronics is the fabrication of electronic components on flexible polymer substrates. Two driving forces are cost reduction and new integration possibilities into shapes and objects of our daily surroundings [1].

Cost reduction arises from the use of polymer materials that are inexpensive compared to standard silicon. Polymerscan be fabricated in high volumes and are casted into "endless" films. These films can be processed in roll-toroll (R2R) fabrication using i.e. cheap printing technologies to create electronic devices.

R2Rfabrication processes are mainly driven by RFID, solar cell and OLED manufacturing. First gas sensors have been made on endless R2R processes [5]. Substrate materials used for flexible gas sensors are mostly made of polymers like PET/PEN [2] or PI [3]but also paper [4].

The substrate materials that exist differ in physical properties and area of application. PET/PEN are cheap polymers that are transparent, while their heat stability allows only a maximum temperature of $150^{\circ} \mathrm{C}$. PI has a better heat stability and can be used up to $300^{\circ} \mathrm{C}$ but is not transparent. Both materials are common for gas sensors, while PET/PEN is more applied with displays and OLEDS and PI with flexible circuits.
Gas sensor applications require special needs depending on the working principle of the sensor and the sensor material [8].

In case of metal oxides as gas sensitive material the substrate has to be heated during operation of sensors. This requires temperatures above the melting point of PET/PEN. In case of capacitive sensors the substrate material should be inert against environmental changes so that the signal is only dependent on the gas sensitive layer.

$\mathrm{PI}$ has a high water adsorption that affects the sensing performance [6]. Bare $\mathrm{PI}$ and PEN substrates have been used for capacitive humidity sensors. Compared to PI, PEN/PET have a lower water absorption coefficient and are less affected from humidity changes [7].

The effect on the gas sensor performance has not been measured, especially resistive type gas sensors such as carbon black composite gas sensors lack in substrate comparison.

In this paper we compare different substrate materials used for gas sensors. We fabricated resistive type polymer composite gas sensors on different polymeric substrates (PI, PEN, PPS and $\mathrm{PEI}$ ) and measured the performance of the gas sensors to an exposure of acetone in synthetic air as well as a variation in humidity. As ground truth we fabricated the same sensors on rigid glass substrates that were tested together with the polymeric substrates. 


\section{Materials and Methods}

Polystyrene (PS) was purchased from Scientific Polymer Products. As conductive filler EnsacoG350 (Timcal) was used. As flexible substrate material PEN Teonex (Du-Pont Tejin films), PI Kapton EN (Du-Pont), PPS Torelina (Toray) and PEI Ultem (Ajedium) was used. All substrates had a thickness of $50 \mu \mathrm{m}$.

In a glass bottle with $30 \mathrm{ml}$ of solvent $4 \mathrm{wt} \%$ of polymer was added under continuous stirring and completely dissolved. To the polymer solution $15 \mathrm{wt} \%$ (of the polymer weight) of carbon black was added. The solution was sonicated using a $500 \mathrm{~W}$ probe sonicator (Sonics Vibra-cell VCX-500) to homogenously disperse the particles. The sonication was done at $150 \mathrm{~W}$ for $6 \mathrm{~min}$ in pulsed mode having $2 \mathrm{~s}$ sonication and $1 \mathrm{~s}$ pause. To prevent the solution from heating up and thus strong solvent evaporation we ran the process in an ice-bath and kept the solution temperature below $30^{\circ} \mathrm{C}$.

Sensor measurements are done using a multi switch measure unit (Agilent 34980A).

In an exsiccator (2I volume), the sensors were exposed to analytes (water and acetone) room temperature. The exsiccator was flushed with $1000 \mathrm{sccm}$ of synthetic air (80\% N2 5.0 and $20 \%$ O2 5.0) using a Voegtlin mass flow controller. For humidity testing water was bubbled with a certain percentage of the total flow through a bottle containing pure DI water and measured its response for $10 \mathrm{~min}$. The flow was set to achieve a humidity level between $0 \% \mathrm{RH}$ and $70 \% \mathrm{RH}$. After the exposure to water we measured the sensor for $10 \mathrm{~min}$ in pure synthetic air to recover. Measurements with acetone as analyte were done using a bottled gas standard containing a calibrated mixture of synthetic air (as before) plus 1000 ppm acetone

With a temperature and humidity sensor (SHT11 Sensirion) we controlled the temperature and humidity inside the chamber during the measurements. The accuracy of the system is in the range of $\pm 3 \% \mathrm{RH}$ and $\pm 1{ }^{\circ} \mathrm{C}$.

All substrates were cleaned with Acetone/IPA and are preshrunk in an oven between 150$200{ }^{\circ} \mathrm{C}$ (depending on $\mathrm{T}_{\mathrm{g}}$ ) for one day. In a second step they were cleaned with an oxygenplasma to promote adhesion. As electrodes and contacts we deposited Ti/Au with a thickness of $5 / 100 \mathrm{~nm}$ using a Plassys thermal evaporator and patterned it using a standard lift-off technique.

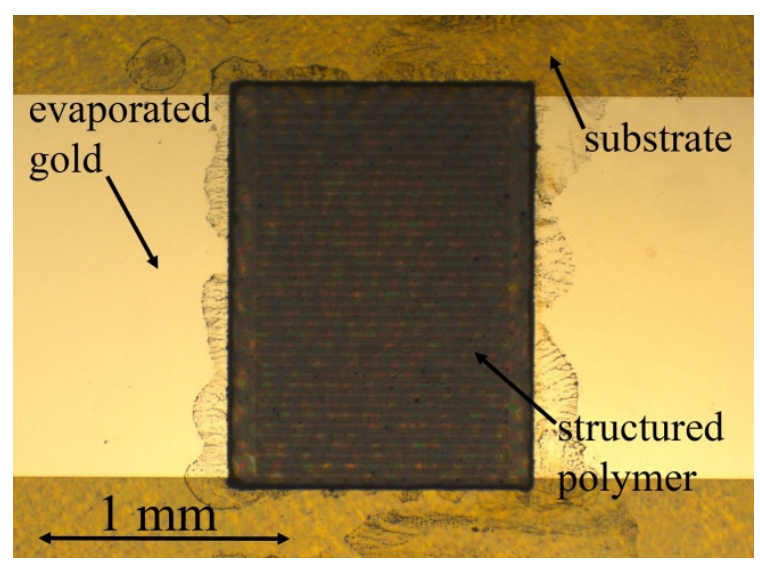

Fig.1.A micro graph of a fabricated gas sensor using the lift-off technique.

The polymer composite sensing material is deposited by spin coating (2000 rpm with $2 \mathrm{~s}$ acceleration for 40s) and patterned with a lift-off technique. A micro graph of a fabricated gas sensor is shown in Fig. 1.

\section{Results and Discussion}

Substrate properties play an important role already during the gas sensor fabrication. One important first parameteristhe adhesion of the evaporated metal layers to the substrate. Without proper adhesion mechanical stress introduced i.e. by bending could destroy the sensor performance by the formation of cracks in the metal.Scratching tests were performed on evaporated metal structures. Without pretreatment good metal adhesion was found on PI, PPS and PEI all evaporated lines stayed intact. The adhesion was poor on PEN and scratched metal lines were destroyed.

The second parameter we observed was the performance of a gas sensor during exposure to different humidity levels. Humidity has a minor swelling effect on the PS sensing material. This leads to minor sensor resistance changes even during humidity jumps greater than $50 \%$ in relative humidity.

The reason for the insensitivity to water is the difference in solubility parameter between water and PS. Only analytes that have a similar solubility parameter as the polymer are able to swell or even dissolve the polymer.

In Fig. 2 the effect of different humidity levels is shown on the normalized resistance for all substrate materials. The humidity is linearly increased in six cycles from about 0 to $60 \% \mathrm{RH}$. Between each cycle the chamber is flushed with dry air. 


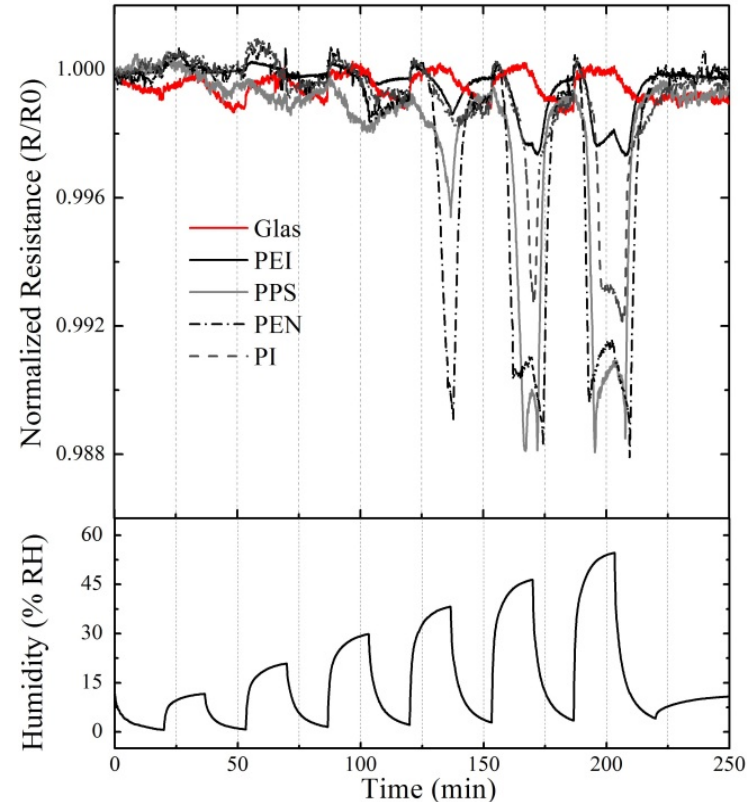

Fig. 2 Influence of variation in humidity on the normalized resistance of gas sensors on different substrate materials.

As ground truth, the sensor on the glass substrate (red line) showed a minor resistance influence (below $0.1 \%$ )upon changes in humidity levels until $60 \% \mathrm{RH}$.

The sensor resistance increased with an increase in humidity. With a decrease in humidity the sensor resistance decreased and settled at the initial resistance value.

Depending on the polymer substrate material the humidity influences are more distinct. From 0 to $30 \% \mathrm{RH}$ the change in resistance is below $0.2 \%$ forthe various gas sensors on polymer substrates. They behaved similar like sensors on glass substrates. At a change from 0 to 40 $\%$ RH PEN substrates showed a significant resistance change of about $1 \%$. Also PPS substrates showed a resistance change of about $0.5 \%$. This trend increased with greaterchanges in humidity levels. The change in sensor resistance was delayed to the actual change in humidity. It was delayed with about 2 min from the change in humidity. This gives rise thatthe reason for the resistance change could be a slower diffusion process in the substrate. PI substrates show a resistance change larger than $0.5 \%$ only at a jump in humidity from $50 \%$. PEI substrates are the most stable and show at a jump from $60 \% \mathrm{RH}$ a resistance change below $0.2 \%$.

Fig. 3 depicts the gas sensor response on all substrates to a repeated exposure of $500 \mathrm{ppm}$ Acetone.

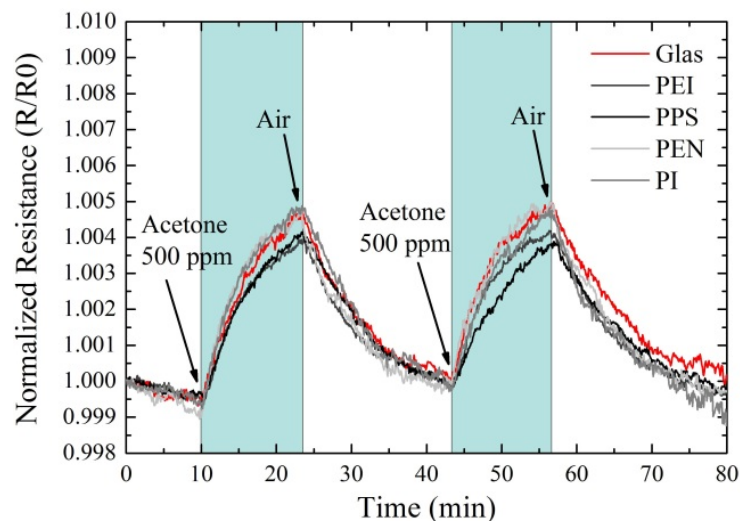

Fig. 3 Influence of 500 ppm acetone exposure to the normalized resistance of gas sensors on different substrate materials.

The gas sensors on all substrate materials show nearly the same behavior to the analyte exposure of $500 \mathrm{ppm}$ acetone. The sensor on glass (red line) acts again as ground truth. The change in normalized resistance was about $0.5 \%$. This change was lower than the change introduced by a jump in humidity from $0 \% \mathrm{RH}$ to $50 \%$ RH for PEN, PPS and PI substrates.

In Tab. 1some material properties of the substrate are shown. One property related to humidity is the coefficient of hydroscopic expansion (CHE).This is a measure of the expansion of a substrate due to humidity uptake of the substrate. The data was only available for PI and PPS and does not agree with the data from Fig. 2.

On water uptake the substrate should increase in size and simultaneously with this expansion also the resistance should increase. In Fig.2 the resistance of the sensors decreased with a delay of 2 min to the increase of humidity and increased with a delay of $2 \mathrm{~min}$ to the decrease of humidity. Another effect that leads to the decrease of sensor resistance must be present that is not known to the authors yet.

Another property is the water absorption of the substrate material. This is the weight of water that a substrate can store in percentage of its own weight. The water absorption for PPS is the lowest compared to the other substrate materials. In Fig.2 PPS does not show lower influence on resistance during the exposure to humidity, even if the value for water absorption is one order of magnitude lower than the other materials.

Water absorptiondoes not play a significant role on resistive polymer gas sensors as it is not a measure for substrate expansion. It is more important for capacitive gas sensors as the dielectric constant of the substrate changes due to water absorption. 
Tab. 1: Properties of substrate materials

\begin{tabular}{|c|c|c|c|c|}
\hline & PEN & PI & PPS & PEI \\
\hline $\mathrm{T}_{\mathrm{G}}$ & $121{ }^{\circ} \mathrm{C}$ & $354{ }^{\circ} \mathrm{C}$ & $92{ }^{\circ} \mathrm{C}$ & $217{ }^{\circ} \mathrm{C}$ \\
\hline $\mathrm{T}_{\mathrm{M}}$ & $269{ }^{\circ} \mathrm{C}$ & - & $285{ }^{\circ} \mathrm{C}$ & - \\
\hline $\mathrm{CTE}$ & $18 \mathrm{ppm} /{ }^{\circ} \mathrm{C}$ & $16 \mathrm{ppm} /{ }^{\circ} \mathrm{C}$ & $30 \mathrm{ppm} /{ }^{\circ} \mathrm{C}$ & $31 \mathrm{ppm} /{ }^{\circ} \mathrm{C}$ \\
\hline Solvent Stability & ++ & ++ & ++ & ++ \\
\hline Transparency & transparent & - & translucent & translucent \\
\hline CHE & n.a. & $8 \mathrm{ppm} / \% \mathrm{RH}$ & $1.5 \mathrm{ppm} / \% \mathrm{RH}$ & n.a. \\
\hline Water Absorption & $0.3 \%$ & $1.8 \%$ & $0.05 \%$ & $0.2 \%$ \\
\hline
\end{tabular}

\section{Conclusion}

We tested different polymerfilms (PEN, PI, PPS and PEI) to their performance as substrates for gas sensors. As ground truth we compared the gas sensors on polymer films with the same gas sensor on a glass substrate. The influence of water on the gas sensor performance showed least influences with PEI as substrate materials.

The exposure to acetone did not show a significant difference in performance of the various substrate materials used.

Besides the stable measurement results with PEI substrates, the polymer combines several benefits of the other substrates. It has low water absorption, is translucent and can be heated up to $200{ }^{\circ} \mathrm{C}$ without degradation (soldering is possible). Further the evaporation of metal films on the substrate showed excellent adhesion already on non-plasma treated substrates. These properties make PEI a promising substrate material for resistive type gas sensor applications.

\section{Acknowledgements}

This work has been scientifically evaluated by the Swiss National Scientific Foundation (SNSF), and financed by the Swiss Confederation and Nano-Tera.ch.

\section{References}

[1] A. Dodabalapur, A.C. Arias, C.D. Frisbie, D. Gamota, T.J. Marks, C. Wood, European Research and Development in Hybrid Flexible Electronics, WTEC Panel Report, July 2010

[2] P.G. Su, C.T. Lee, and C.Y. Chou. Flexible NH3 sensors fabricated by in situ self-assembly of polypyrrole. Talanta 80(2), 763-769 (2009); doi:10.1016/j.talanta.2009.07.057
[3] E. Abad, S. Zampolli, S. Marco, A. Scorzoni, B. Mazzolai, A. Juarros, D. G'omez, I. Elmi, G.C. Cardinali, J.M. G'omez, et al. Flexible tag microlab development: gas sensors integration in rfid flexible tags for food logistic. Sensors and Actuators B: Chemical, 127(1), 2-7(2007); doi:10.1016/j.snb.2007.07.007

[4] A. Arena, N. Donato, G. Saitta, A. Bonavita, G. Rizzo, and G. Neri, Flexible ethanol sensors on glossy paper substrates operating at room temperature, Sensors and Actuators B: Chemical, 145(1), 488-494 doi:10.1016/j.snb.2009.12.053

(2010);

[5] Radislav A. Potyrailo, Andrew Burns, Cheryl Surman, D. J. Lee and Edward McGinniss,Multivariable passive RFID vapor sensors: roll-to-roll fabrication on a flexible substrate, (2012)doi:10.1039/C2AN16278D

Analyst,

[6] A. Oprea, N. Barsan, U. Weimar, J. Courbat, D. Briand, N.F. de Rooij, Integrated Temperature, Humidity and Gas Sensors on Flexible Substrates for Low-Power Applications, Proceedings IEEE Sensors, 158-161 (2007); doi:10.1109/ICSENS.2007.4388360

[7] A. Oprea, N. Barsan, U. Weimar, M. Bauersfeld, D. Ebling, J. Wöllenstein, Capacitive humidity sensors on flexible RFID labels, Sensors and Actuators B 132, 404-410(2008); doi:10.1016/j.snb.2007.10.010

[8] G. Korotcenkov, Chemical Sensors: Comprehensive Sensor Technologies, Vol 6, Chemical Sensors Applications (Sensors Technology Series), Momentum Press (2011), 